\title{
Gap and Van Hove Measurements via Low-loss Electron Energy Loss Spectroscopy on Atomically thin $M_{\mathrm{x}} \mathrm{W}_{(1-\mathrm{x})} \mathrm{S}_{2}$ Nanoflakes
}

\author{
Mario Pelaez Fernandez, Kazu Suenaga, Raul Arenal
}

\begin{abstract}
Band gap tailoring of 2D materials has been of interest for the past years. Using low-loss electron energy loss spectroscopy (EELS), in a scanning transmission electron microscope (STEM), it has been confirmed, following previous experimental (photoluminescence) and theoretical (DFT) studies, that the band gap of atomically thin nanoflakes of $M_{x} W_{(1-x)} S_{2}$ does shift with the alloying degree of the sample.
\end{abstract}

\section{INTRODUCTION}

Atomically thin two-dimensional (2D) materials have been a clear point of interest for research, especially since the discovery of graphene in 2004[1]. This vast interest comes mainly from the attractive electronic, thermal and mechanic properties (among others) that this kind of materials can display; as well as a vast number of potential applications [2]. Within 2D materials, layered transition metal dichalcogenide (TMD) semiconductors of the $\mathrm{TX}_{2}$ type $(\mathrm{T}=\mathrm{Mo}, \mathrm{W}$, etc; $\mathrm{X}=\mathrm{S}$, $\mathrm{Se}, \mathrm{Te}$ ) are graphene-like materials that have attracted an important amount of research interest.

Furthermore, regarding the electronic properties of these 2D materials, the tuning of the band gap is of great importance for the development of more versatile materials for electronic applications. Among others, a commonly used technique for band gap tuning in bulk materials has been the alloying of materials with different band gaps. Up until now, the only atomically thin 2D alloys that have been reported for TMDs have been $\mathrm{MoS}_{2(1-\mathrm{x})} \mathrm{Se}_{2 \mathrm{x}}$ [3] and $\mathrm{Mo}_{\mathrm{x}} \mathrm{W}_{(1-\mathrm{x})} \mathrm{S}_{2}$ [4]. Our study is focused on the latter ones, where both theoretical calculations (DFT), as well as photoluminescence experimental results show that, for monolayers, there is an evidence of band gap tuning changing the alloying degree. Low-loss spatially resolved EELS offers the option of studying the behavior of materials at the nanoscale, allowing not only the study of different properties for different alloying degrees, but also for different stacks of layers within the same sample.

* Research supported by the EU project ESTEEM2 (Integrated Infrastructure Initiative - I3, Grant Agreement 312483), the Spanish MINECO (FIS2013-46159-C3-3-P) and European Union H2020 programs ETN project "Enabling Excellence" Grant Agreement 642742 and "Graphene Flagship" Grant Agreement 696656. Low-loss EELS studies were developed at the Advanced Microscopy Laboratory (LMA) of Institute of Nanoscience of Aragon (INA) - U. of Zaragoza (Spain)

M. Pelaez Fernandez is with the Institute of Nanoscience of Aragon, in Zaragoza, Spain. (e-mail: mariopf@unizar.es)

K. Suenaga is with the Nanotube Research Center, National Institute of Advanced Industrial Science and Technology (AIST), in Tsukuba, Japan (e-mail: suenaga-kazu@aist.go.jp)

R. Arenal is with the Institute of Nanosciences of Aragon in Zaragoza, as well as the ARAID Foundation, in Zaragoza (email: arenal@unizar.es)

\section{EXPERIMENTAL SETUP}

For this anaylsis, several $\mathrm{Mo}_{\mathrm{x}} \mathrm{W}_{(1-\mathrm{x})} \mathrm{S}_{2}$ single crystals showing different alloying degrees $(\mathrm{x}=0,0.3,0.5,0.7$ and 1$)$ were grown by chemical vapor transport as shown in the bibliography [5]. These crystals were first exfoliated using tape and then the flakes obtained were transferred onto tem grids for the experiment.

The microscope taking part in all the spectroscopic analyses performed on this study has been a FEI Titan microscope with an aberration-corrected probe, working at 80 $\mathrm{KV}$. The microscope is equipped as well with a monochromator, which provides an energy resolution of $\sim 180 \mathrm{meV}$.

The alloying degree of the different samples has been directly determined from the ratio of Mo and $\mathrm{W}$ in high-angle annular dark field (HAADF)-STEM images, as the ones shown in Figure 1. It is possible to deduce this ratio based on the different contrasts both elements have in HAADF due to their different atomic densities [3].

For each of the aforementioned samples, areas where a low number of layers could be seen were selected and identified using optical and low-magnification TEM images such as the ones presented in Figure 1. In these regions, several spectra have been taken using the spectrum-line

a)
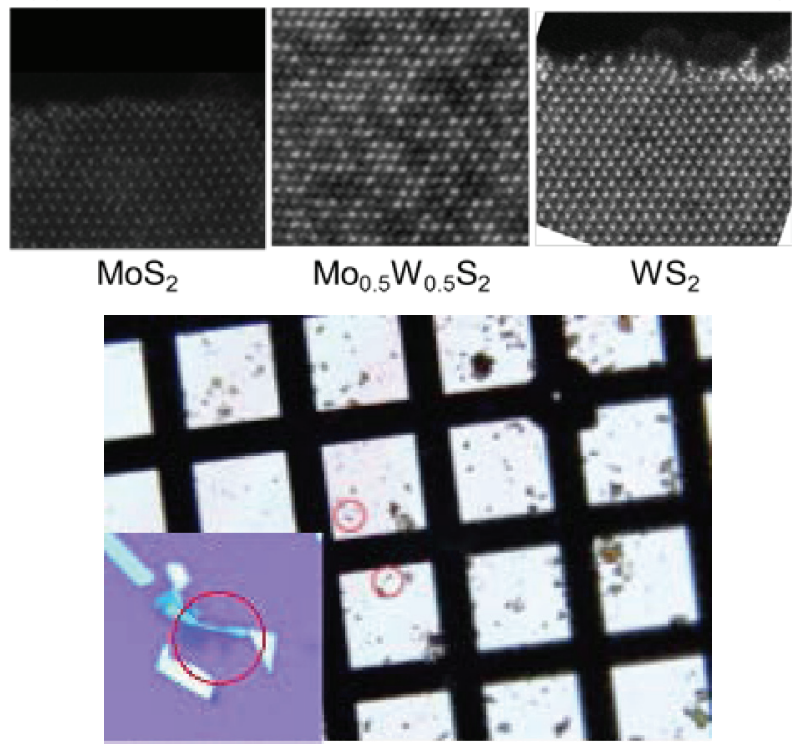

Figure 1: a) Set of three micrographs corresponding to three different samples, showing how the alloying degree of the samples can be obtained. b) Optical characterization and identification of low layer zones. 
technique.

Using the intensity profile in each spectrum-line, zones showing the same number of layers have been found (highlighted flat areas in figure 1.d) and several spectra have been integrated over a window between 5 and $30 \mathrm{~nm}$. The resulting spectra, after zero loss peak (ZLP) removal, can be seen in figure 1.e. In these resulting, different features have been studied for different alloying degrees and number of layers: On the one hand, the band gap feature, occurring in a region between 1.5 and $2 \mathrm{eV}$ altogether with the A,B exciton transition peaks; and on the other hand, two peaks already related in the literature with Van Hove singularities for $\mathrm{MoS}_{2}$ [6]

\section{RESULTS}

Results can be seen in figure 3 . The results concerning the band gap zone (Figure 3.a) show a relation between the average gap of each sample and the alloying degree of the sample. However, there has not been any clear proof of a correlation between the number of layers within each sample and the band gap behavior. On the other hand, the results regarding the Van Hove singularities (Figure 3.b and 3.c) show that, even though apart from the $\mathrm{MoS}_{2}$ there is no evident shift of these peaks with the alloying degree, this shift is clearly visible for different layer stackings within the same sample.
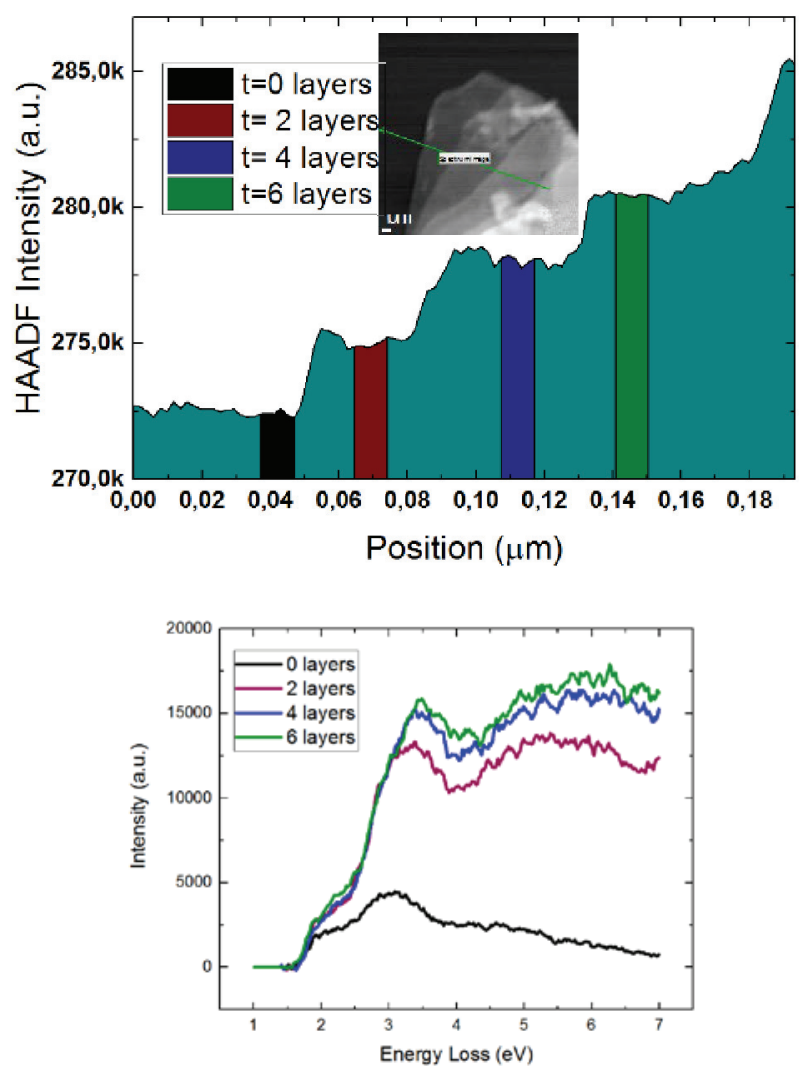

Figure 2: a) Example of a spectrum profile in

$M_{0.5} W_{0.5} S_{2}$. The image shows a micrograph of the chosen flake and its intensity profile, highlighting the regions where spectra have been integrated. b) Integrated spectra resulting from section $a$.

\section{CONCLUSION}

a)
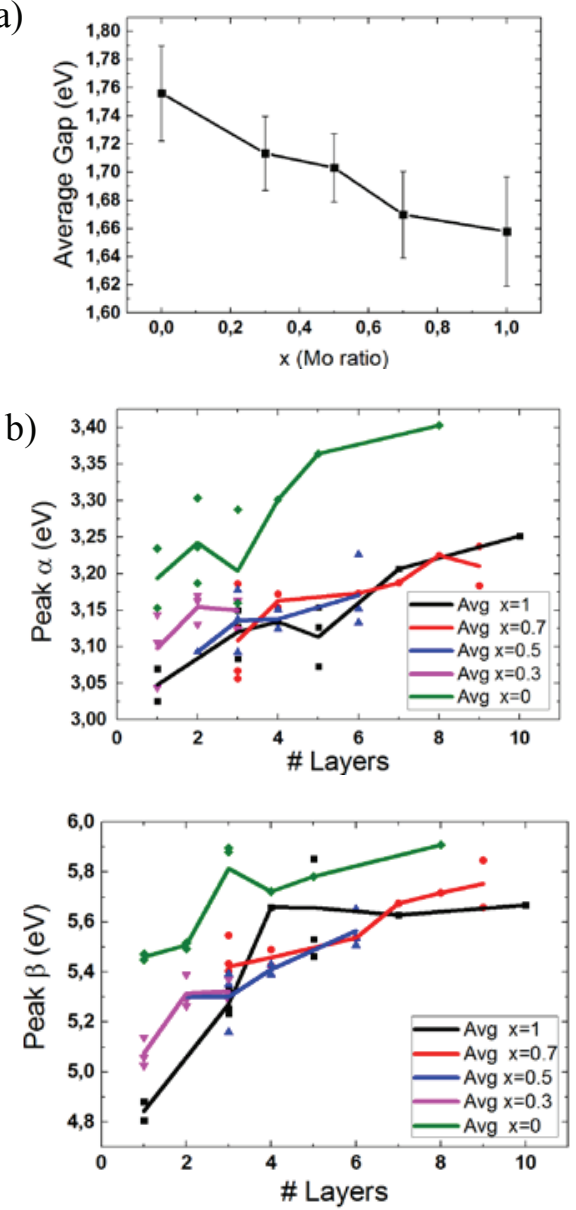

Figure 3: a) Average gap measured for different samples with varying alloying degree. $b$ ) Behavior of the $\alpha$ (top) and $\beta$ (bottom) peaks with the alloying degree and the number of layers.

To sum up, this study improves our knowledge of the optoelectronic properties of atomically thin layered alloys of dichalcogenides and delves deeper into the potential applications these materials could have.

\section{REFERENCES}

[1] Novoselov, K. S.; Geim, et al. Science 2004, 306, 666-669.

[2] Novoselov, K. S.; Geim, A. K. The Rise of graphene. Nature Materials 6, 183 - 191 (2007)

[3] Chen, Y et al (2013). Oral : Tunable Band Gap in Atomically Thin Transition-Metal Dichalcogenide Alloys, (5), 3656.

[4] Feng, Q. et al, Growth of Large-Area 2D MoS 2(1- x) $\mathrm{Se}_{2 \times}$ Semiconductor Alloys. Adv. Mater. 2014, 26, 2648-2653

[5] Dumcenco, D. O.; et al. Raman Study of 2H-Mo1xWxS2 Layered Mixed Crystals. J. Alloys Compd. 2010, 506, 940-943.

[6] Dileep, K. et al, Journal of Applied Physics 119, 114309 (2016); doi: $10.1063 / 1.4944431$

[7] R. Arenal, O. Stephan, Chap. 3, Eds. L. Francis, A. Mayoral, and R. Arenal, Springer (2015).

[8] R. Arenal, et al. Phys. Rev. Lett. 95 (12), 127601 